\title{
Review Article \\ Microelectrical Discharge Machining: A Suitable Process for Machining Ceramics
}

\author{
Andreas Schubert, Henning Zeidler, Ralf Kühn, and Matthias Hackert-Oschätzchen
}

Technische Universität Chemnitz, Professorship Micromanufacturing Technology, 09107 Chemnitz, Germany

Correspondence should be addressed to Henning Zeidler; henning.zeidler@mb.tu-chemnitz.de

Received 30 June 2015; Accepted 16 August 2015

Academic Editor: Young-Wook Kim

Copyright ( 2015 Andreas Schubert et al. This is an open access article distributed under the Creative Commons Attribution License, which permits unrestricted use, distribution, and reproduction in any medium, provided the original work is properly cited.

Today ceramics are used in many industrial applications, for example, in the biomedical field, for high-temperature components or for cutting tools. This is attributed to their excellent mechanical and physical properties, as low density, high strength, and hardness or chemical resistance. However, these specific mechanical properties lead to problems regarding the postprocessing of ceramics. In particular, cutting processes require expensive tools which cause high manufacturing costs to machine ceramics. Consequently, there is a demand for alternative machining processes. Microelectrical discharge machining (micro-EDM) is a thermal abrasion process which is based on electrical discharges between a tool and a workpiece. The advantages of micro-EDM are more and more in focus for ceramic machining. These advantages include the process of being a noncontact technology, an independency of material brittleness and hardness, a low impact on the material, and the achievable microstructures. This paper presents the current state of investigations regarding micro-EDM of ceramics. Beside the process principle of EDM, the used procedures for machining ceramics and insulating ceramics are described. Furthermore several machining examples are presented to demonstrate the possibilities of the micro-EDM process with regard to the machining of ceramics.

\section{Introduction}

In the last decades the interest for using high-performance ceramics steadily increased. This can be attributed to their outstanding properties such as a low density, high hardness and strength even at high temperatures, their chemical resistance, and their wear resistance. Because of this, there is a large area of application for these materials. But, especially for the machining of ceramics, the excellent mechanical properties lead to very high demands with regard to suitable manufacturing processes. Possible postprocessing-in the hardened state-by cutting processes is only possible to a limited extent and requires specific and expensive tools. One possible solution is the near-net-shape fabrication of ceramics. However, in consequence of the shrinkage of ceramics within the sintering process, it is hard to realise functional dimensions. Because of this, postprocessing often is unavoidable for the fabrication of precision parts.

One approach for a solution is the use of abrasive processes like laser machining $[1,2]$ or electrical discharge machining (EDM). Due to their removal of material by a thermal ablation, they are independent of the mechanical properties such as hardness, strength, and brittleness. With the ongoing miniaturization of mechanical systems, there is a demand for small ceramic components. This can be fulfilled by the use of microelectrical discharge machining (microEDM) for a finish machining of ceramic parts.

This paper provides an overview of developments and procedures which allow machining of ceramics by microEDM. Furthermore, selected machining examples are presented which demonstrate the possibilities of this manufacturing process.

\section{Process Principle of Electrical Discharge Machining}

Electrical discharge machining is a process which is based on electrical discharges between two electrodes, the tool and the workpiece. The ablation of the material is realised 

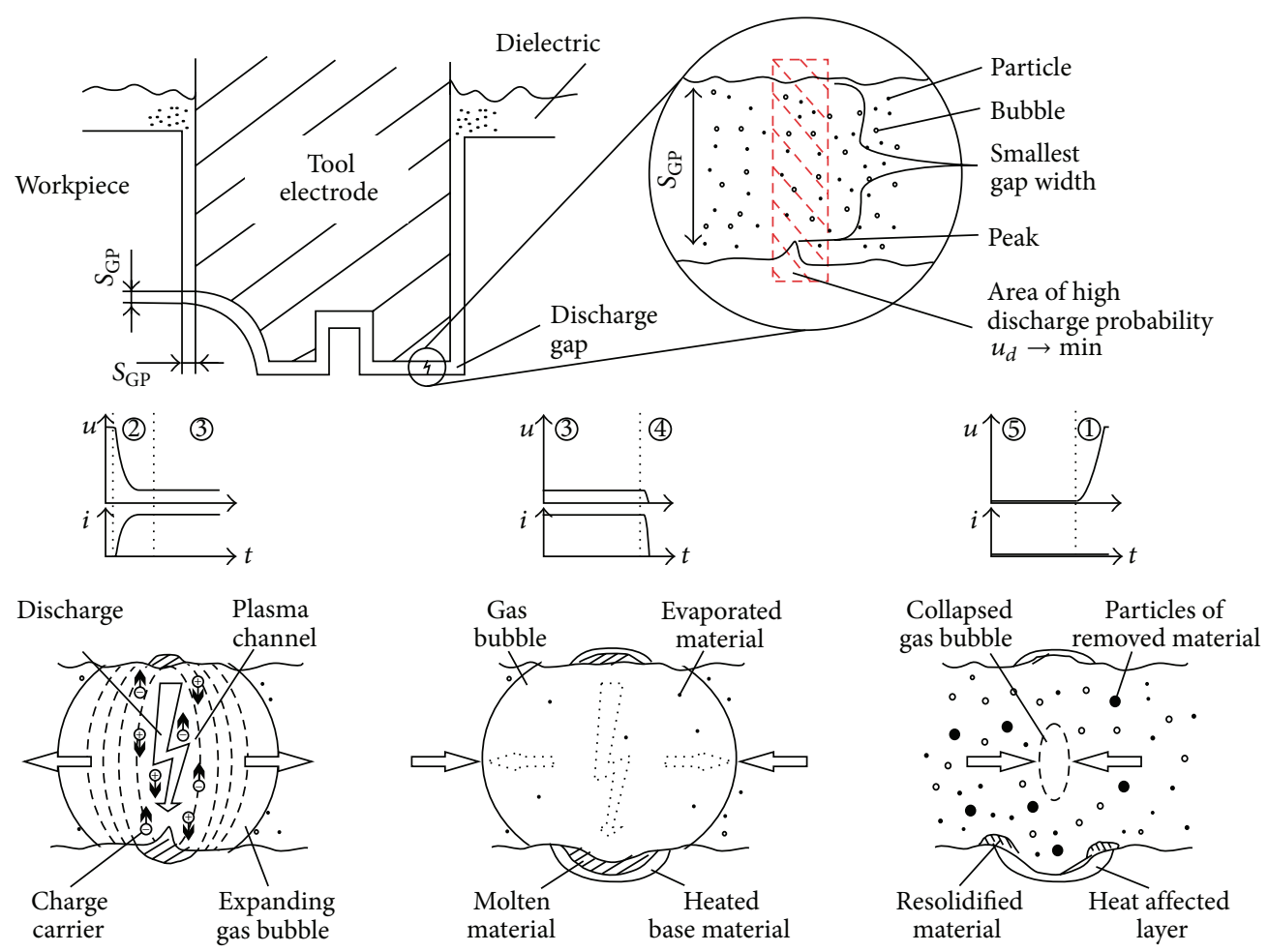

FIGURE 1: Process principle of electrical discharge machining [19].

by melting and evaporation of material. Figure 1 shows the process principle.

Tool and workpiece are separated by a dielectric medium, either dielectric oil or deionised water. For realising an electrical discharge, a voltage is applied between tool and workpiece and the distance between the electrodes is decreased. By reaching the breakdown voltage of the dielectric medium, a discharge takes place, establishing a plasma channel which causes a current flow. Thus, temperatures of more than $10000 \mathrm{~K}$ occur and the material gets melted and evaporated, creating an expanding gas bubble.

Stopping the input of the energy leads to a collapse of the plasma channel and therefore to an end of the discharge. The formed gas bubble collapses, too. The particles of the removed material are flushed away by this and the flow of the dielectric medium. By applying a voltage between tool and workpiece again, the process repeats in the same procedure.

A repetition of this process leads to a removal of material and due to the tool feed in direction of the workpiece its shape is resembled in the material. Through the thermal influence on both electrodes, there is also a wear at the tool. The resulting surface can be described by an assembly of single discharges which lead to a crater-like topology. The dimension of the craters strongly depends on the used process energy which can be modified by selecting respective process parameters.

The discharges take place at the site of the lowest dielectric breakdown voltage. Usually, this is the area of the smallest distance between both electrodes. But due to presence of particles of removed material and gas bubbles, it is possible that the breakdown voltage of the dielectric fluid is reduced by these, which leads to discharges in areas with a higher distance between tool and workpiece.

This noncontact process behaviour is the main benefit of electrical discharge machining. Due to the thermal ablation of the material, it is independent of material hardness and brittleness. Furthermore, by the nearly force-free processing, it is possible to use thin and fragile tool electrodes to realise small geometries with high aspect ratios.

In micro-EDM, usually discharge durations of $t_{e} \leq 1 \mu \mathrm{s}$ and discharge energies of $W_{e} \leq 100 \mu \mathrm{J}$ are realised. It is possible to achieve craters with a diameter of $\leq 5 \mu \mathrm{m}$ and a depth of $\leq 1 \mu \mathrm{m}$. This leads to resulting surface roughness lower than $R_{\mathrm{z}} \leq 1 \mu \mathrm{m}$.

\section{Electrical Discharge Machining of Ceramics}

Electrical discharge machining requires a minimum electric conductivity about $10^{-2} \Omega^{-1} \mathrm{~cm}^{-1}$. This demand is fulfilled by metallic materials. The minimum electric conductivity constitutes a critical factor for the possibility of electrical discharge machining of ceramics. As an overview, Figure 2 shows the electrical conductivity for selected metallic materials and ceramics.

Electrically conductive ceramics like silicon doped silicon carbide $(\mathrm{SiSiC})$, titanium diboride $\left(\mathrm{TiB}_{2}\right)$, or titanium nitride (TiN) can be machined by EDM $[3,4]$ like metallic materials. For nonconductive ceramics like $\mathrm{ZrO}_{2}$ (zirconia), aluminium oxide $\left(\mathrm{Al}_{2} \mathrm{O}_{3}\right)$, or silicon nitride $\left(\mathrm{Si}_{3} \mathrm{~N}_{4}\right)$, the electric conductivity is too low to achieve an electrical discharge between 


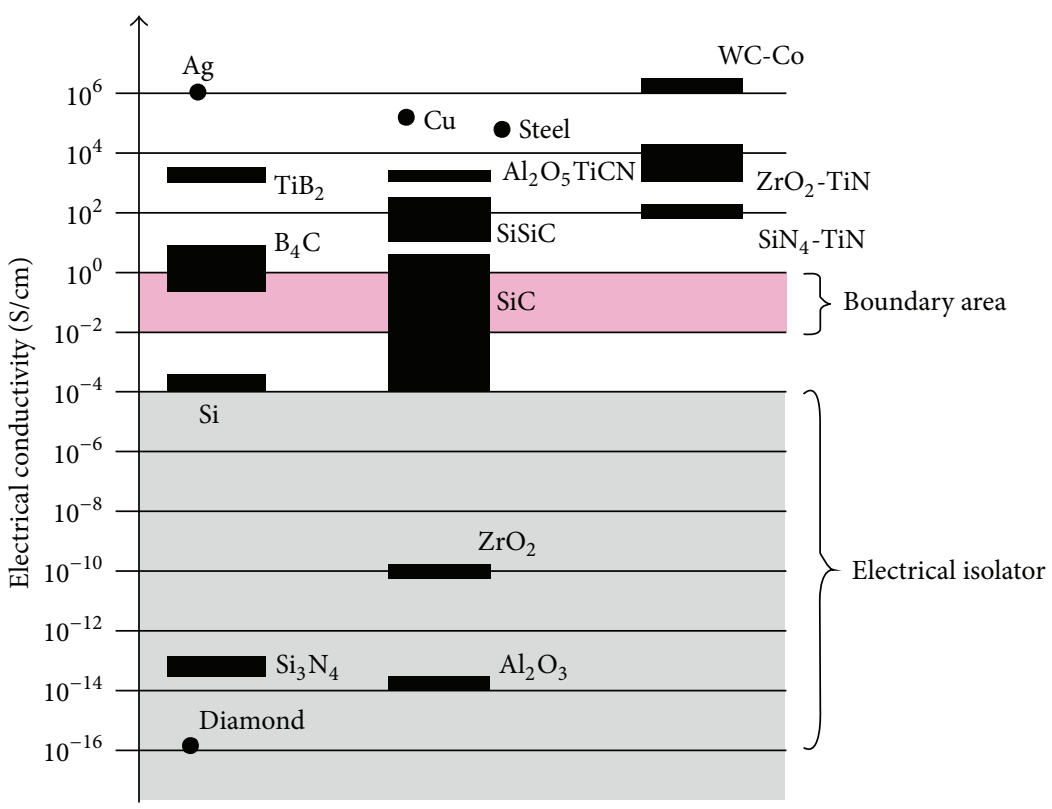

FigURE 2: Overview of materials and their electrical conductivity [3].

tool and workpiece and therefore using a conventional EDM process is not possible. However, there are two different possibilities which allow machining of nonconductive ceramics. The first option is a doping of the ceramics by a conductive phase. Thus, it is possible to increase the conductivity above $10^{-2} \Omega^{-1} \mathrm{~cm}^{-1}$ which enables machining of these ceramics by EDM. The second option is the so-called "assisting electrode method." For this procedure, an electrical conductive starting layer is used to begin the process.

3.1. Doped Ceramics. As already mentioned, it is possible to influence the conductivity of insulating ceramics like $\mathrm{ZrO}_{2}$, $\mathrm{Al}_{2} \mathrm{O}_{3}$, and $\mathrm{Si}_{3} \mathrm{~N}_{4}$ by doping. To achieve this, a secondary electrically conductive phase is incorporated into the material which enables the possibility to machine these ceramics by EDM. Successful approaches were achieved with the reinforcements of $\mathrm{TiB}_{2}, \mathrm{WC}, \mathrm{ZrB}_{2}, \mathrm{TiC}, \mathrm{TiCN}$, and $\mathrm{TiN}$ in zirconia [5-7]. Yoo et al. also used yttrium nitrate (YN) for realising an electrically conductive $\mathrm{SiC}$ ceramic [8]. For $\mathrm{Al}_{2} \mathrm{O}_{3}$, the incorporating with $\mathrm{TiN}$ and $\mathrm{TiCN}$ was reported as successful attempt to realise machining by EDM [6, 9]. For $\mathrm{Si}_{3} \mathrm{~N}_{4}$ mainly reinforcements of $\mathrm{TiN}$ were used for doping $[10,11]$.

One disadvantage of this procedure is the influence of these reinforcements on the mechanical properties of the material, which could lead to undesirable effects. By increasing of the content of TiN, a decreasing of the hardness, the fracture toughness, and the flexural strength of $\mathrm{ZrO}_{2}$ can be observed [5].

3.2. Assisting Electrode Method. The "assisting electrode method" for machining insulating ceramics was first mentioned and investigated by Mohri et al. [12, 13]. Figure 3 shows a schematic illustration of the process principle. In this procedure, a conductive layer is applied on the surface of the insulating ceramic. Thereby, the process can initially be started by machining this conductive starting layer. Mohri et al. used a copper plate, respectively, a copper mesh as material for the conductive layer. A hydrocarbon oil was used as dielectric fluid within the experiments.

The procedure is based on the effect that machining of the thin starting layer material also causes an ablation of the workpiece material below. Additionally, the hydrocarbons in dielectric oil are disintegrated and carbon is available. On the ceramic surface, a secondary thin conductive layer is formed which enables a continuous EDM process for these insulating ceramics. By thoroughly controlling this process, it is possible to achieve a stable repetition of this sequence of removing the conductive layer and the underlying workpiece material and the deposition of a secondary layer onto the surface. The machining is possible as long as the conductive layer can be generated reliably. By using deionised water, the assisting electrode could not be realised yet.

The formation of a conductive layer during the assisting electrode method also leads to a different shape of the discharge pulses. Figure 4 shows exemplarily a comparison of a discharge for metallic materials and for the insulating ceramic zirconia.

The discharge pulses were realised with the same process parameters. For both graphs, the voltage $U$ is illustrated by the blue curve and the current by the red line. The typical "metal" discharge in micro-EDM can be characterised by a rapid voltage drop with an overshoot into the negative range and a simultaneous rise of the current (in this case to $2 \mathrm{~A}$ ). The duration of the discharge is in the range of hundred nanoseconds. After the discharge, the generator capacitor is immediately recharged, which can be recognized by the rising voltage. In micro-EDM of insulating ceramics like $\mathrm{ZrO}_{2}$, a different pulse shape can be observed. Compared to the 


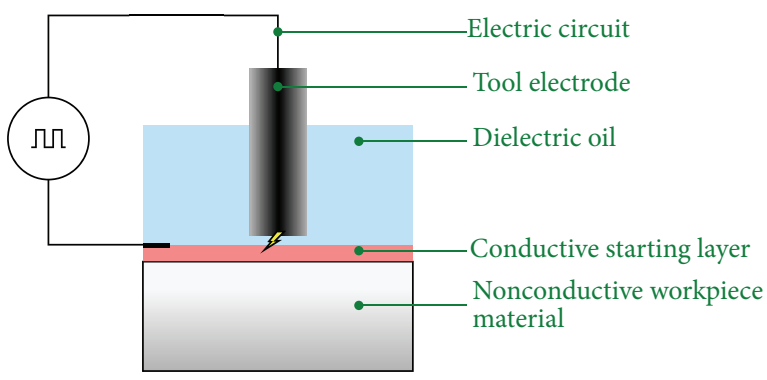

(a)

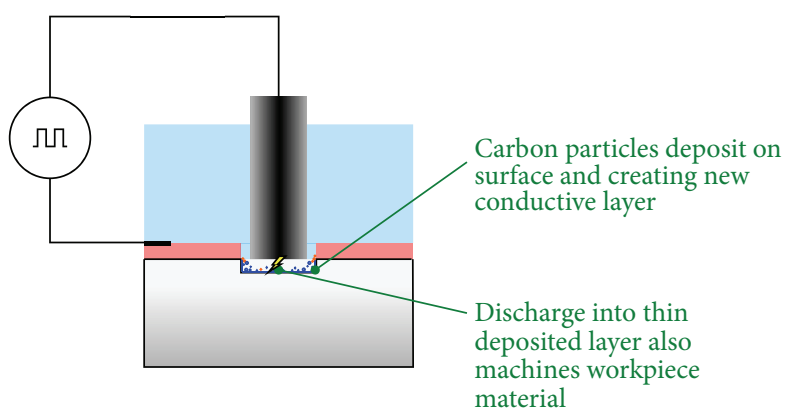

(c)

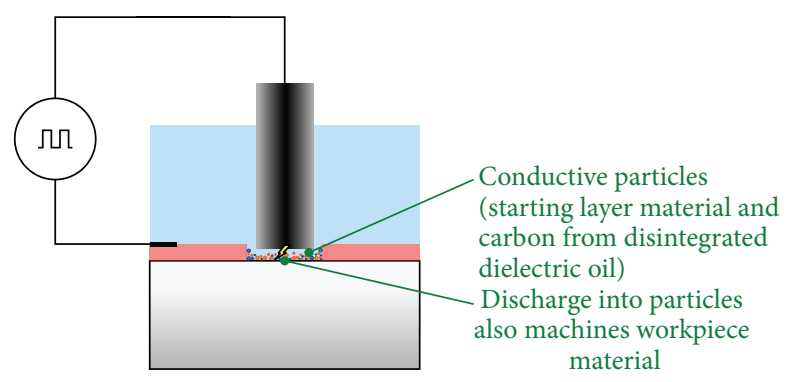

(b)

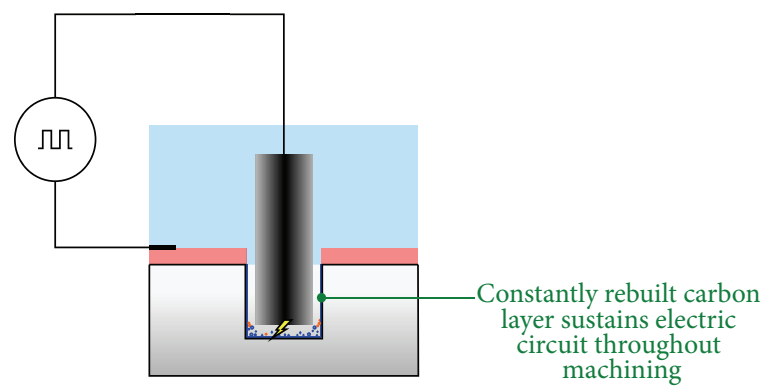

(d)

FIGURE 3: Assisting electrode scheme for machining nonconductive ceramic materials [19].

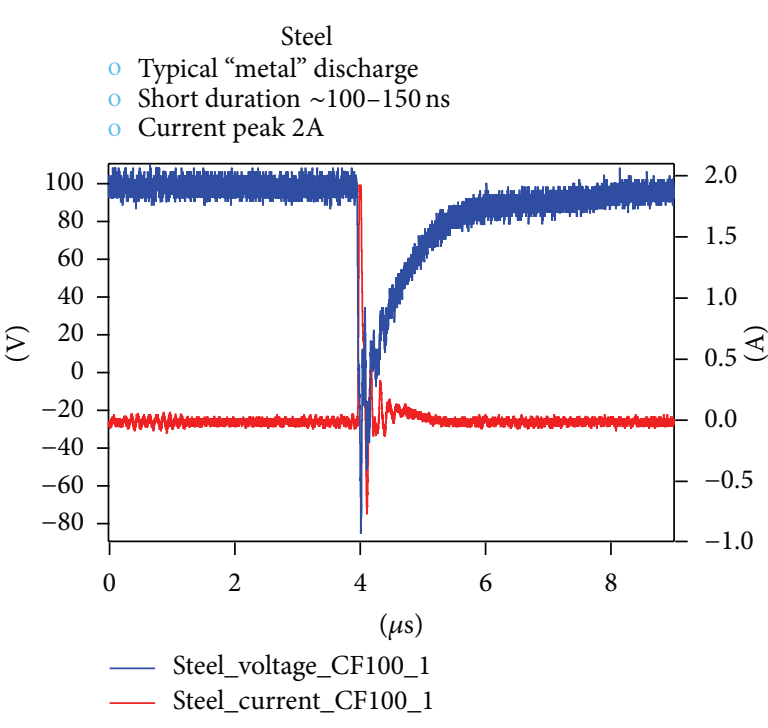

(a)

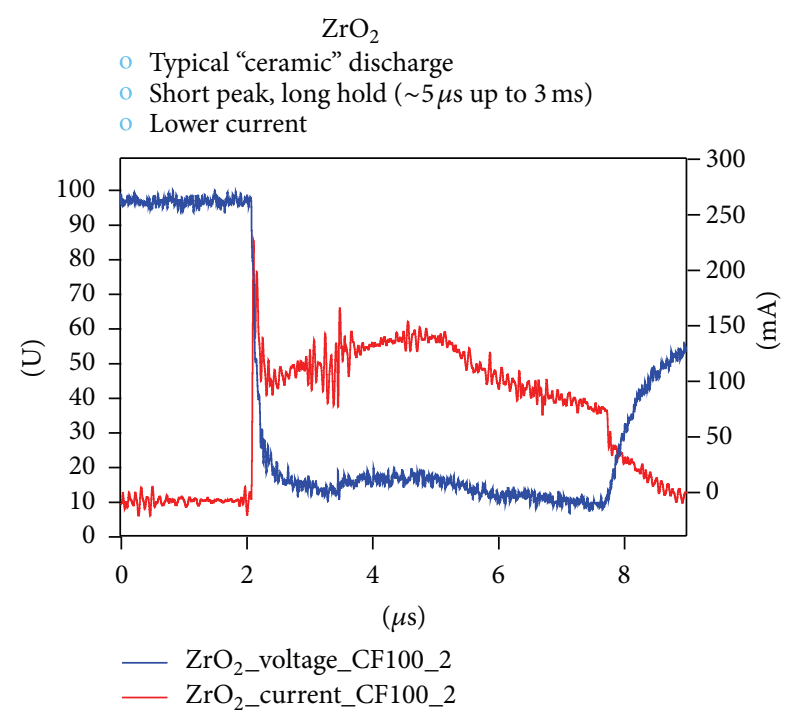

(b)

FIgURE 4: Comparison of a single discharge for metal (a) and for insulating ceramic zirconia (b) in micro-EDM.

typical "metal" discharge, there is a much slower voltage drop to approximately $15 \mathrm{~V}$ without overshoot into the negative range. At the beginning of the discharge, there is a short current peak similar to those in metal but with a lower current value of about $0.2 \mathrm{~A}$. This is thought to be attributed to the much lower electric conductivity of the formed conductive layer in comparison with metals. For example, Mohri et al. estimated a specific resistance of $8.1 * 10^{-2} \Omega \mathrm{cm}$ for the formed layer on $\mathrm{Si}_{3} \mathrm{~N}_{4}$ [14], which is much lower than the values for steel (about $1 * 10^{-5} \Omega \mathrm{cm}$ to $2 * 10^{-5} \Omega \mathrm{cm}$ ).
Following this, there is a second phase of the discharge characterised by a longer hold of the current and voltage at a specific value which cannot be observed for typical "metal" discharges. In this example, the duration of this phase is about $6 \mu$ s. Subsequently, the current decrease to zero and the used RC pulse generator is charged which can be observed by the rising voltage. Fukuzawa et al. and Schubert and Zeidler report that the electrically conductive layer is built trough disintegration of the hydrocarbon based EDM oil during this long period $[15,16]$. 


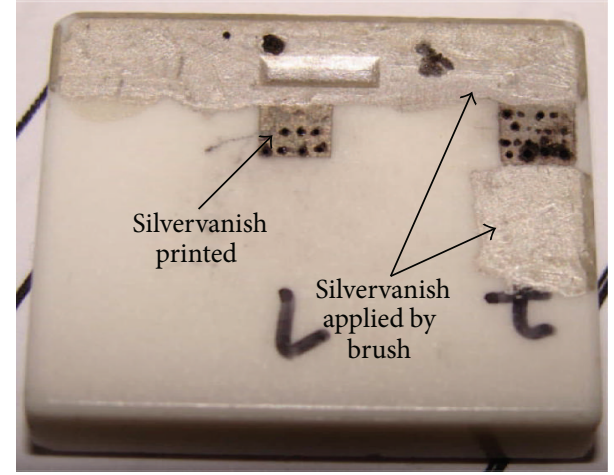

FIGURE 5: $\mathrm{ZrO}_{2}$-sample with coatings of conductive silver lacquer, printed or applied by brush.

3.3. Current Research for EDM of Insulating Ceramics. Several studies were done to investigate the assisting electrode method for improving the applicability of this procedure. Key topics are the ablation behaviour of the single insulating ceramics $\left(\mathrm{ZrO}_{2}, \mathrm{Al}_{2} \mathrm{O}_{3}, \mathrm{Si}_{3} \mathrm{~N}_{4}\right.$, and $\left.\mathrm{SiC}\right)$, the used materials for the starting layer, and the layer generation process itself.

For $\mathrm{ZrO}_{2}$, it is suggested that the rebuilt conductive layer is $\mathrm{ZrC}[13,17]$. The deposition of $\mathrm{ZrC}$ could also be observed for a new developed $\mathrm{ZrB}_{2}$-Cu tool electrode [18]. In contrast to the ceramic $\mathrm{Al}_{2} \mathrm{O}_{3}$, machining of $\mathrm{Si}_{3} \mathrm{~N}_{4}, \mathrm{SiC}$, and $\mathrm{ZrO}_{2}$ has been done successfully by using the assisting electrode method $[15,19-25]$. For $\mathrm{Al}_{2} \mathrm{O}_{3}$ machining by EDM is more critical because the creation of the conductive layer depends upon the degree of purity of the material [20]. Mohri et al. report that due to the higher free forming energy of $\mathrm{Al}_{4} \mathrm{C}_{3}$ compared to those of $\mathrm{ZrC}$ and $\mathrm{TiC}$ the generation of a conductive layer is more difficult on $\mathrm{Al}_{2} \mathrm{O}_{3}$ which leads to a more unstable process $[13,26]$.

There are several different procedures for the preparation of a conductive layer which were realised and investigated. Mohri et al. used a TiN-layer deposited by a physical vapour deposition process (PVD) [14]. Sabur et al. and Banu et al. used a copper foil as starting layer for the assisting electrode method [20,22]. Another possibility is the usage of conductive silver lacquer $[16,27]$ which can be printed and sintered or applied by brush. Mohri et al. also used a method called "baked-carbon" to realise a starting layer $[13,26]$. The most important requirement is the mechanical attachment of the conductive layer onto the workpiece surface. For example, Schubert and Zeidler reported no useable results by using foils in combination with a vibration of the workpiece [27]. Figure 5 shows exemplarily a sample of $\mathrm{ZrO}_{2}$ with a conductive silver lacquer which is usually used at the Technische Universität Chemnitz for experimental investigations in micro-EDM of insulating ceramics.

Main requirement for machining insulating ceramics by micro-EDM is a stable process which is based on continuous forming of a conductive layer on the surface of the workpiece. To achieve this, the process parameters have to be optimized. Crucial is especially the transition moment from ablating the assisting electrode to first creation and machining of the secondary layer. Here, suitable feed to discharge ratio has

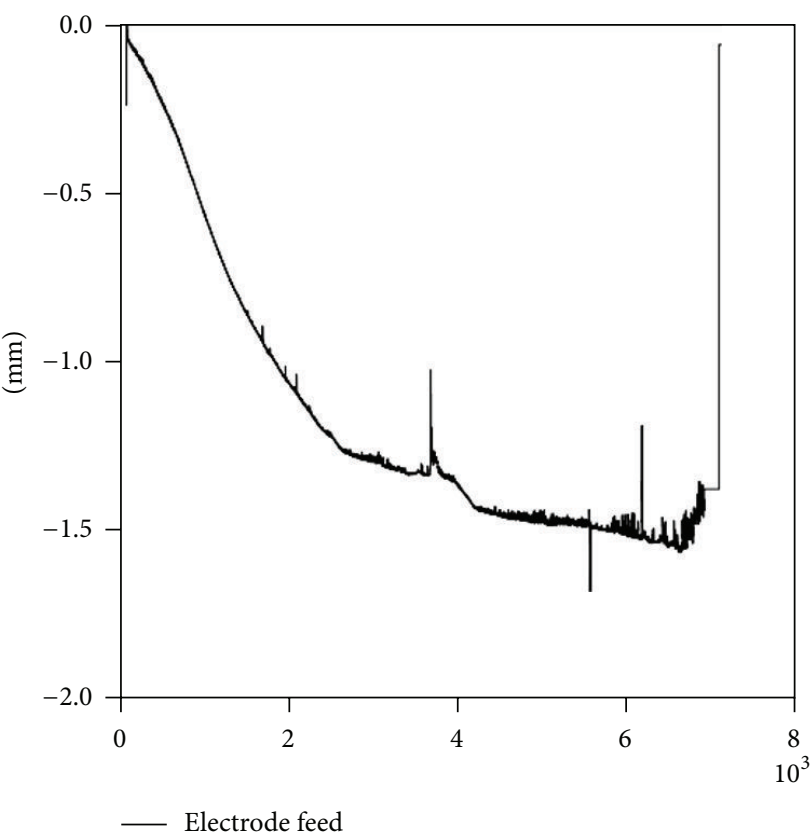

FIGURE 6: Recording of the feed while machining microbores in $\mathrm{ZrO}_{2}[16]$.

to be implemented in order to avoid tool breakage if the secondary layer is not yet sufficiently formed. Furthermore, for high aspect ratio bores, the changing electrical boundary conditions have to be considered. Schubert and Zeidler reported a decreasing current up to $50 \%$ by increasing machining depth for machining $\mathrm{ZrO}_{2}$ [16]. This is attributable to a rising electrical resistance with growing bore depth. Due to this, the process speed declines until the feed rate approaches zero. This behaviour can be observed in Figure 6 .

The rising electrical resistance causes decreasing of the current and the discharge energy. It has to be assumed that the discharge energy falls below a threshold value which is necessary to create efficient discharges and thus remove workpiece material.

Another important influencing parameter for micromachining of insulating ceramics is the flushing. Schubert et al. reported an improvement in processing time, bore depth, and tool wear by using a rotating tool and vibration [28]. Due to the this, there is better flushing and thus better removing of debris. Banu et al. could only realize stable machining by using a rotational speed between $300 \mathrm{rpm}$ and $600 \mathrm{rpm}$ [22].

By means of these examples, it is clear that main goal for research is the optimisation of process parameters for achieving a stable process which is marked by stable forming of the conductive layer.

\section{Machining Examples for Micro-EDM of Ceramics}

In this chapter selected examples are presented which illustrate the possibilities in micro-EDM of ceramics.

4.1. Doped Ceramics. Ferraris et al. realised an extrusion die and an air bearing component by using a micro-EDM 

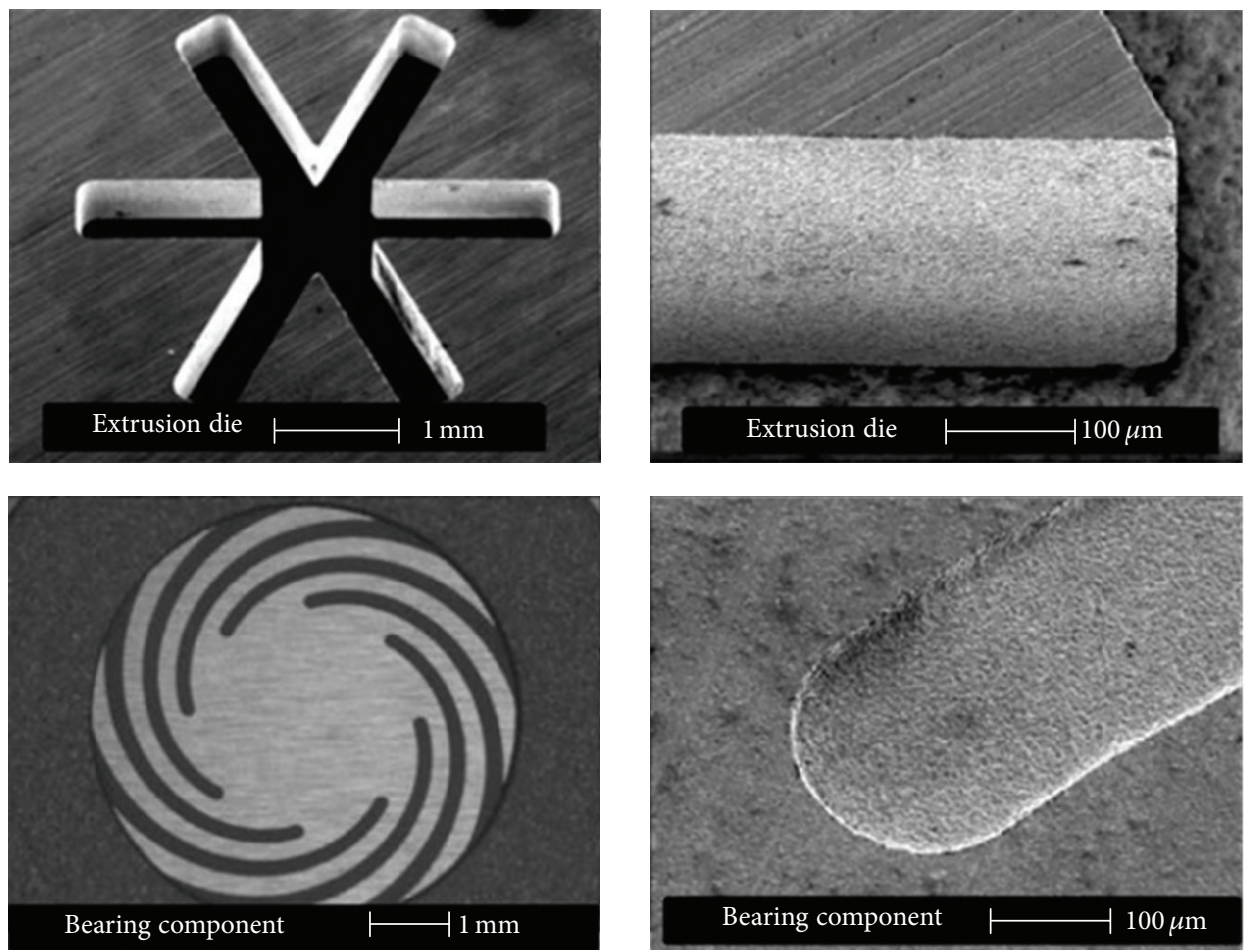

FIgURE 7: SEM pictures and particulars of the $\mathrm{Al}_{3} \mathrm{O}_{2}-\mathrm{TiCN}$ extrusion die and $\mathrm{ZrO}_{2}-\mathrm{TiN}$ air bearing component [6].
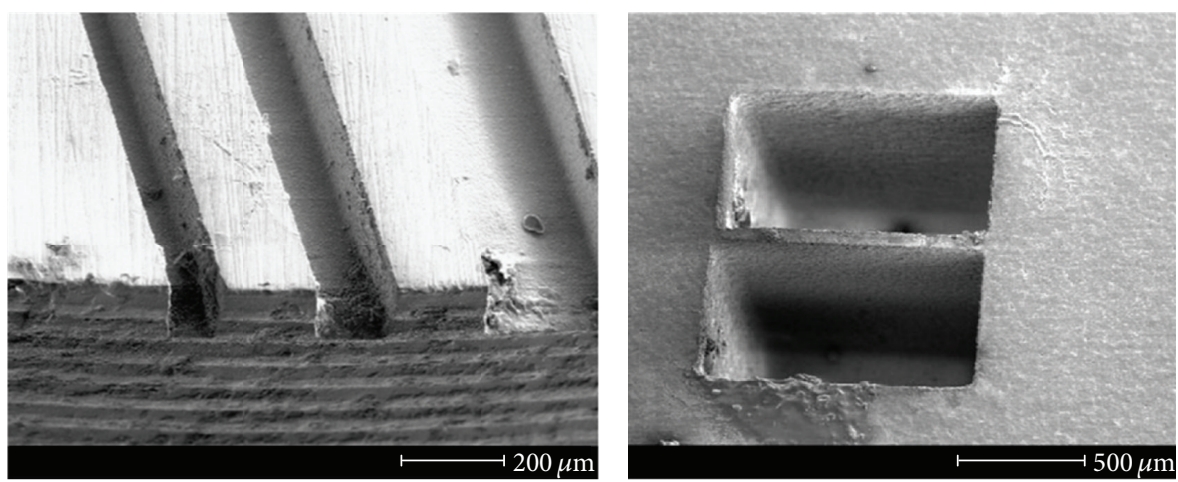

FIGURE 8: SEM image of channel geometries [21] and a complete bar made with a $100 \mu \mathrm{m}$ distance plate in $\mathrm{ZrO}_{2}$ [29].

milling strategy for ceramic composites $\mathrm{Al}_{3} \mathrm{O}_{2}-\mathrm{TiCN}$ and $\mathrm{ZrO}_{2}-\mathrm{TiN}[6]$. Figure 7 shows SEM images of the fabricated components.

The star shaped deep drawing tool has a diameter of $3 \mathrm{~mm}$ which can be used for fibre optic extrusion. The second part is a thrust air bearing surface which consists of $0.015 \mathrm{~mm}$ deep circular grooves. Both parts were realised by a layer-by-layer milling strategy with a machining time of $1 \mathrm{~h} \mathrm{[6].}$

4.2. Insulating Ceramics. Hösel et al. realised different channel geometries in zirconia by using a structured copper tool electrode [21]. Figure 8, left, shows a SEM image of the machined grooves.

The grooves were fabricated by sinking the structured copper electrode into the zirconia sample. The channel geometries had a length of $4 \mathrm{~mm}$ with widths of $0.5 \mathrm{~mm}$, $0.25 \mathrm{~mm}, 0.125 \mathrm{~mm}$, and $0.08 \mathrm{~mm}$ at a depth of $0.2 \mathrm{~mm}$. Hösel et al. also machined rigid bars in $\mathrm{ZrO}_{2}$ by using a modular tool concept [29]. Figure 8, right, shows a SEM image of a complete bar machined with a distance plate of $100 \mu \mathrm{m}$. By this procedure, it was possible to erode bars with aspect ratios of more than 80 . They achieved bars with heights of $1.5 \mathrm{~mm}$ and for the smallest bar a width of $8 \mu \mathrm{m}$.

Several experimental investigations regarding the microEDM of insulating ceramics were performed at the Technische Universität Chemnitz by using two Sarix micro-EDM machines. One of them, a Sarix Sx-100 HPM, is shown in Figure 9.

Amongst other parts, Schubert and Zeidler realised microbores and pole structures in zirconia [16]. Figure 10, 


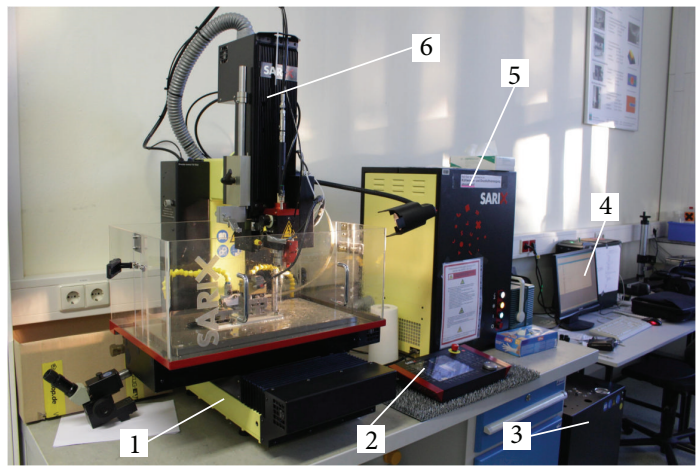

FIgure 9: Photo of the used Sarix SX-100 HPM micro-EDM milling machine at the Technische Universität Chemnitz. (1) Machine frame, (2) control system, (3) dielectric fluid aggregate, (4) computer, (5) power supply, and (6) pulse generator.
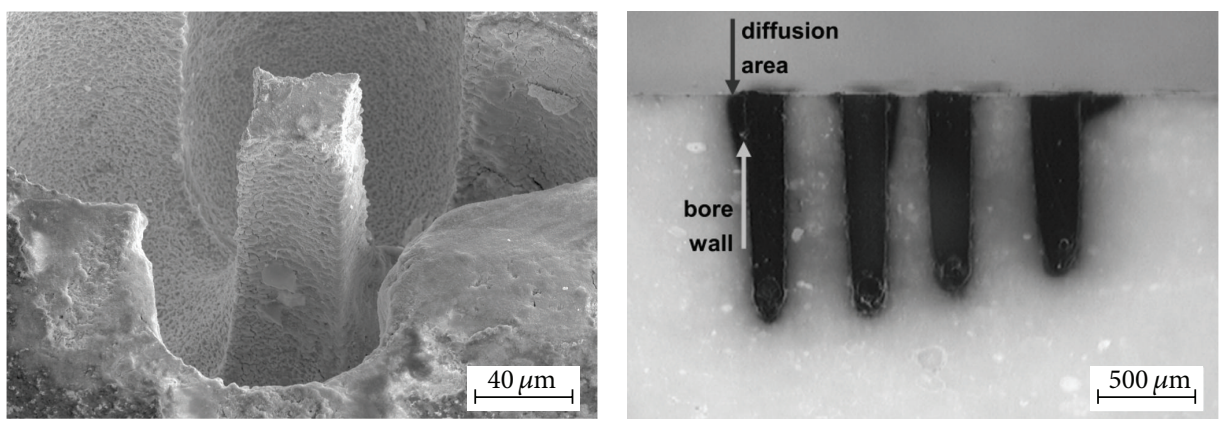

FIGURE 10: SEM image of a pole structure machined in $\mathrm{ZrO}_{2}$ [16] and cross section of microbores in $\mathrm{ZrO}_{2}$ with strong diffusion of carbide into the base material [19].
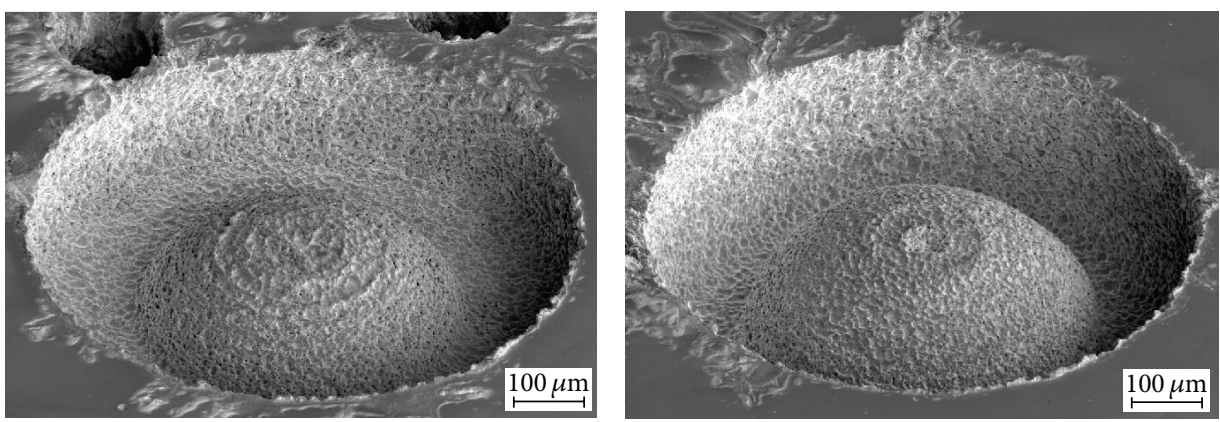

FIGURE 11: SEM images of two hemispheres in zirconia made by micro-EDM milling with different parameter settings.

left and right, shows a SEM image of the feature. The pole structure with a width of $50 \mu \mathrm{m}$ was realised by the subsequent machining of four microbores. Schubert and Zeidler also realised microbores with a diameter of less than $200 \mu \mathrm{m}$ and aspect ratios of 5.8. Higher aspect ratios for these microbores could not be achieved due to an insufficient material removal. Figure 10, right, shows a cross section of the realised microbores. A strong diffusion of carbide into the base material can be observed within the bores.

In further experiments, at the Technische Universität Chemnitz, the machining of complex geometries in zirconia was investigated. Two fabricated hemispheres in $\mathrm{ZrO}_{2}$ are illustrated in Figure 11.

Both of the hemispheres were realised by a layer-bylayer micro-EDM milling process with different process parameters. The diameter of the hemisphere is about $500 \mu \mathrm{m}$. As can be seen in the SEM-images, there is a difference in the surface quality and the precision. This demonstrates that it is necessary to know the ablation behaviour of insulating ceramics for realising parts with high precision and accuracy.

However, there is still a strong demand of research regarding the micro-EDM for insulating ceramics concerning 
the understanding of the process, the influence of the process parameters, and the ablation behaviour of the single insulating ceramics.

\section{Summary}

In this paper, the microelectrical discharge machining of ceramics and especially insulating ceramics was presented. This is a field of research which is intensively investigated at the Technische Universität Chemnitz. Beside the characterisation of the ablation behaviour for different ceramics (e.g., $\mathrm{ZrO}_{2}, \mathrm{Si}_{3} \mathrm{~N}_{4}-\mathrm{TiN}$, or alumina toughened zirconia), investigations were conducted regarding the surface roughness, the achievable aspect ratios, and the fabrication of complex structures.

Recent developments, procedures, and machining examples in micro-EDM of ceramics were described and illustrated in this paper.

The results of research show that micro-EDM is a suitable process for postprocessing of ceramics. Due to the assisting electrode method, it is also possible to machine insulating ceramics. There is also a strong demand for further investigations to the micro-EDM of ceramics. Topic areas are the extension of processable ceramic materials, the improvement of the ablation process of special ceramics (e.g., $\mathrm{Al}_{2} \mathrm{O}_{3}$ ), the optimisation of the process behaviour, and a better understanding of the formation of the conductive layer.

\section{Conflict of Interests}

The authors declare that there is no conflict of interests regarding the publication of this paper.

\section{References}

[1] A. N. Samant and N. B. Dahotre, "Three-dimensional laser machining of structural ceramics," Journal of Manufacturing Processes, vol. 12, no. 1, pp. 1-7, 2010.

[2] A. N. Samant and N. B. Dahotre, "Laser machining of structural ceramics-a review," Journal of the European Ceramic Society, vol. 29, no. 6, pp. 969-993, 2009.

[3] K. Liu, B. Lauwers, and D. Reynaerts, "Crossing barriers in structuring ceramics," Microniek, vol. 50, no. 1, pp. 28-34, 2010.

[4] A. Schoth, R. Förster, and W. Menz, "Micro wire EDM for high aspect ratio 3D microstructuring of ceramics and metals," Microsystem Technologies, vol. 11, no. 4-5, pp. 250-253, 2005.

[5] S. Salehi, O. Van der Biest, and J. Vleugels, "Electrically conductive $\mathrm{ZrO}_{2}$-TiN composites," Journal of the European Ceramic Society, vol. 26, no. 15, pp. 3173-3179, 2006.

[6] E. Ferraris, D. Reynaerts, and B. Lauwers, "Micro-EDM process investigation and comparison performance of $\mathrm{Al}_{3} \mathrm{O}_{2}$ and $\mathrm{ZrO}_{2}$ based ceramic composites," CIRP Annals-Manufacturing Technology, vol. 60, no. 1, pp. 235-238, 2011.

[7] J. Vleugels and O. Van der Biest, "Development and characterization of $\mathrm{Y}_{2} \mathrm{O}_{3}$-stabilized $\mathrm{ZrO}_{2}$ (Y-TZP) composites with $\mathrm{TiB}_{2}, \mathrm{TiN}, \mathrm{TiC}$, and $\mathrm{TiC}_{0.5} \mathrm{~N}_{0.5}$," Journal of the American Ceramic Society, vol. 82, no. 10, pp. 2717-2720, 1999.

[8] H.-K. Yoo, J.-H. Ko, K.-Y. Lim, W. T. Kwon, and Y.-W. Kim, "Micro-electrical discharge machining characteristics of newly developed conductive SiC ceramic," Ceramics International, vol. 41, no. 3, pp. 3490-3496, 2015.

[9] B. Lauwers, J. P. Kruth, W. Liu, W. Eeraerts, B. Schacht, and P. Bleys, "Investigation of material removal mechanisms in EDM of composite ceramic materials," Journal of Materials Processing Technology, vol. 149, no. 1-3, pp. 347-352, 2004.

[10] K. Liu, E. Ferraris, J. Peirs, B. Lauwers, and D. Reynaerts, "Micro-EDM process investigation of $\mathrm{Si}_{3} \mathrm{~N}_{4}$-TiN ceramic composites for the development of micro-fuel-based power units," International Journal of Manufacturing Research, vol. 3, no. 1, pp. 27-47, 2008.

[11] K. Liu, J. Peirs, and E. Ferraris, "Micro electrical discharge machining of $\mathrm{Si}_{3} \mathrm{~N}_{4}$-based ceramic composites," in Proceedings of the 4th International Conference on Multi-Material Micro Manufacture, pp. 161-166, September 2008.

[12] N. Mohri, Y. Fukuzawa, T. Tani, N. Saito, and K. Furutani, "Assisting electrode method for machining insulating ceramics," CIRP Annals-Manufacturing Technology, vol. 45, no. 1, pp. 201-204, 1996.

[13] N. Mohri, Y. Fukuzawa, T. Tani, and T. Sata, "Some considerations to machining characteristics of insulating ceramics-towards practical use in industry," CIRP AnnalsManufacturing Technology, vol. 51, no. 1, pp. 161-164, 2002.

[14] N. Mohri, Y. Fukusima, Y. Fukuzawa, T. Tani, and N. Saito, "Layer generation process on work-piece in electrical discharge machining," CIRP Annals-Manufacturing Technology, vol. 52, no. 1, pp. 157-160, 2003.

[15] Y. Fukuzawa, N. Mohri, H. Gotoh, and T. Tani, "Threedimensional machining of insulating ceramics materials with electrical discharge machining," Transactions of Nonferrous Metals Society of China (English Edition), vol. 19, no. 1, pp. s150s156, 2009.

[16] A. Schubert and H. Zeidler, "Implementation of Micro-EDM process for machining of nonconductive ceramics," in Proceedings of the 16th International Symposium on Electromachining, pp. 565-569, April 2010.

[17] A. Schubert, H. Zeidler, M. Hahn, M. Hackert-Oschätzchen, and J. Schneider, "Micro-EDM milling of electrically nonconducting zirconia ceramics," Procedia CIRP, vol. 6, pp. 297-302, 2013.

[18] A. K. Khanra, B. R. Sarkar, B. Bhattacharya, L. C. Pathak, and M. M. Godkhindi, "Performance of $\mathrm{ZrB} 2-\mathrm{Cu}$ composite as an EDM electrode," Journal of Materials Processing Technology, vol. 183, no. 1, pp. 122-126, 2007.

[19] A. Schubert, H. Zeidler, M. H. Oschätzchen, J. Schneider, and M. Hahn, "Enhancing micro-EDM using ultrasonic vibration and approaches for machining of nonconducting ceramics," Journal of Mechanical Engineering, vol. 59, no. 3, pp. 156-164, 2013.

[20] A. Sabur, M. Y. Ali, M. A. Maleque, and A. A. Khan, "Investigation of material removal characteristics in EDM of nonconductive $\mathrm{ZrO}_{2}$ ceramic," Procedia Engineering, vol. 56, pp. 696701, 2013.

[21] T. Hösel, C. Müller, and H. Reinecke, "Spark erosive structuring of electrically nonconductive zirconia with an assisting electrode," CIRP Journal of Manufacturing Science and Technology, vol. 4, no. 4, pp. 357-361, 2011.

[22] A. Banu, M. Y. Ali, and M. A. Rahman, "Micro-electro discharge machining of non-conductive zirconia ceramic: investigation of MRR and recast layer hardness," International Journal of Advanced Manufacturing Technology, vol. 75, no. 1-4, pp. 257267, 2014. 
[23] A. Muttamara, Y. Fukuzawa, N. Mohri, and T. Tani, "Probability of precision micro-machining of insulating $\mathrm{Si}_{3} \mathrm{~N}_{4}$ ceramics by EDM," Journal of Materials Processing Technology, vol. 140, no. 1-3, pp. 243-247, 2003.

[24] D. Hanaoka, Y. Fukuzawa, C. Ramirez, P. Miranzo, M. I. Osendi, and M. Belmonte, "Electrical discharge machining of ceramic/ carbon nanostructure composites," Procedia CIRP, vol. 6, pp. 95-100, 2013.

[25] A. Muttamara, P. Janmanee, and Y. Fukuzawa, "A study of micro-EDM on silicon nitride using electrode materials," International Transaction Journal of Engineering, Management, \& Applied Sciences \& Technologies, vol. 1, no. 1, pp. 1-7, 2010.

[26] Y. Fukuzawa, N. Mohri, T. Tani, and A. Muttamara, "Electrical discharge machining properties of noble crystals," Journal of Materials Processing Technology, vol. 149, no. 1-3, pp. 393-397, 2004.

[27] A. Schubert and H. Zeidler, "Machining of nonconductive $\mathrm{ZrO} 2$ ceramics with micro-EDM," in Proceedings of the 9th International Conference of the European Society for Precision Engineering and Nanotechnology (EUSPEN '09), pp. 6-9, June 2009.

[28] A. Schubert, H. Zeidler, N. Wolf, and M. Hackert, "Micro electro discharge machining of electrically nonconductive ceramics," AIP Conference Proceedings, vol. 1353, pp. 1303-1308, 2011.

[29] T. Hösel, P. Cvancara, T. Ganz, C. Müller, and H. Reinecke, "Characterisation of high aspect ratio non-conductive ceramic microstructures made by spark erosion," Microsystem Technologies, vol. 17, no. 2, pp. 313-318, 2011. 

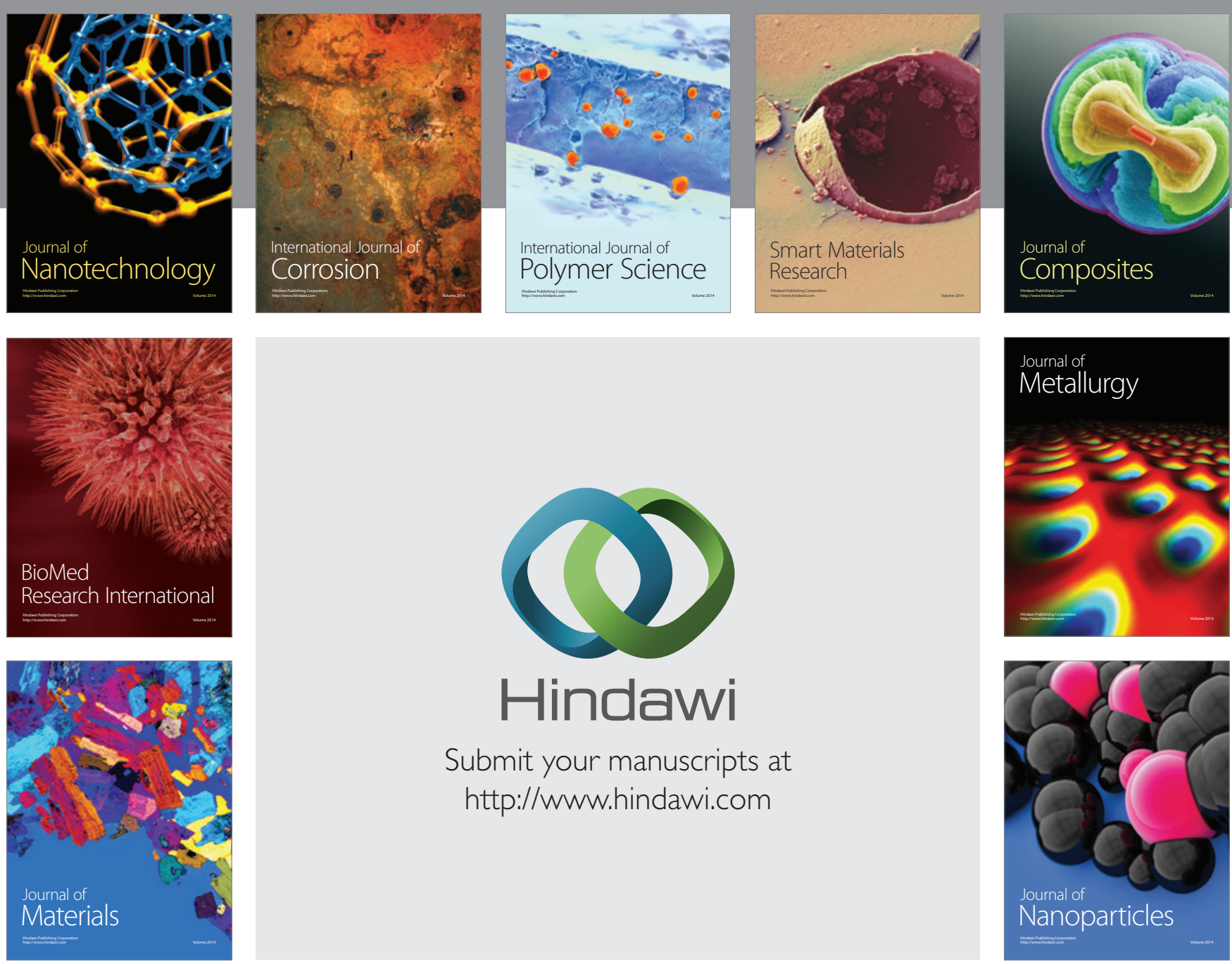

Submit your manuscripts at http://www.hindawi.com
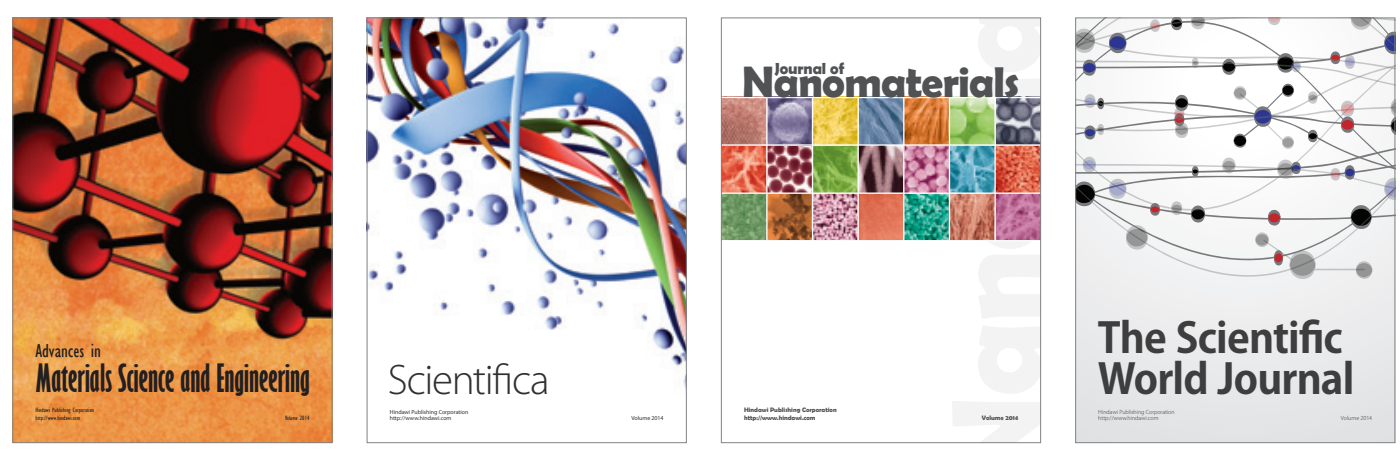

\section{The Scientific World Journal}
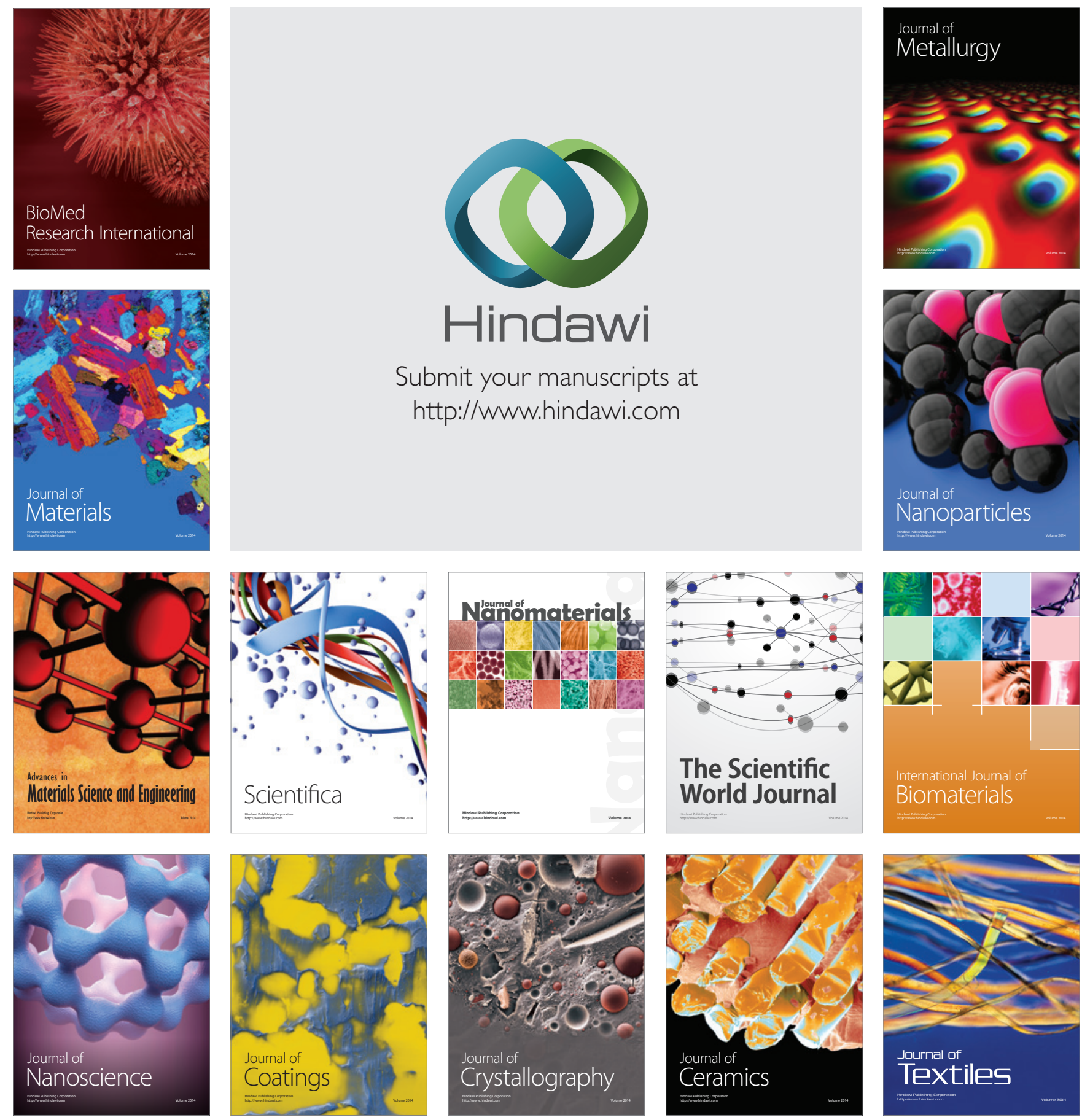